\title{
Life Cycle Dominates the Volatilome Character of Dimorphic Fungus Coccidioides spp.
}

\author{
Emily A. Higgins Keppler, ${ }^{a, b}$ Heather L. Mead, ${ }^{c}$ Bridget M. Barker, (D)Heather D. Bean ${ }^{a, b}$ \\ aSchool of Life Sciences, Arizona State University, Tempe, Arizona, USA \\ bCenter for Fundamental and Applied Microbiomics, The Biodesign Institute, Arizona State University, Tempe, Arizona, USA \\ cPathogen and Microbiome Institute, Northern Arizona University, Flagstaff, Arizona, USA
}

ABSTRACT Valley fever (coccidioidomycosis) is an endemic fungal pneumonia of the North and South American deserts. The causative agents of Valley fever are the dimorphic fungi Coccidioides immitis and C. posadasii, which grow as mycelia in the environment and as spherules within the lungs of vulnerable hosts. Current diagnostics for Valley fever are severely lacking due to poor sensitivity and invasiveness, contributing to a 23-day median time to diagnosis, and therefore, new diagnostic tools are needed. We are working toward the development of a breath-based diagnostic for coccidioidomycosis, and in this initial study, we characterized the volatile metabolomes (or volatilomes) of in vitro cultures of Coccidioides. Using solid-phase microextraction (SPME) and comprehensive two-dimensional gas chromatography coupled to time of flight mass spectrometry (GC $\times$ GC-TOFMS), we characterized the volatile organic compounds (VOCs) produced by six strains of each species during mycelial or spherule growth. We detected a total of 353 VOCs that were at least 2fold more abundant in a Coccidioides culture than in medium controls and found that the volatile metabolome of Coccidioides is more dependent on the growth phase (spherules versus mycelia) than on the species. The volatile profiles of C. immitis and C. posadasii have strong similarities, indicating that a single suite of Valley fever breath biomarkers can be developed to detect both species.

IMPORTANCE Coccidioidomycosis, or Valley fever, causes up to $30 \%$ of communityacquired pneumonias in highly populated areas of the U.S. desert southwest where the disease is endemic. The infection is difficult to diagnose by standard serological and histopathological methods, which delays appropriate treatment. Therefore, we are working toward the development of breath-based diagnostics for Valley fever. In this study, we characterized the volatile metabolomes (or volatilomes) of six strains each of Coccidioides immitis and C. posadasii, the dimorphic fungal species that cause Valley fever. By analyzing the volatilomes during the two modes of growth of the fungus-mycelia and spherules-we observed that the life cycle plays a significant role in the volatiles produced by Coccidioides. In contrast, we observed no significant differences in the $C$. immitis versus $C$. posadasii volatilomes. These data suggest that life cycle, rather than species, should guide the selection of putative biomarkers for a Valley fever breath test.

KEYWORDS GC $\times \mathrm{GC}$, Valley fever, biomarkers, coccidioidomycosis, comprehensive two-dimensional gas chromatography, dimorphic fungus, fungal infections, untargeted metabolomics, volatile metabolites

occidioidomycosis, or Valley fever, is a disease that is endemic to the deserts of the western United States, Mexico, and Central and South America and is responsible for an estimated 350,000 new infections per year (1). In highly populated areas of the
Citation Higgins Keppler EA, Mead HL, Barker BM, Bean HD. 2021. Life cycle dominates the volatilome character of dimorphic fungus Coccidioides spp. mSphere 6:e00040-21. https://doi.org/10.1128/mSphere.00040-21. Editor Aaron P. Mitchell, University of Georgia Copyright $\odot 2021$ Higgins Keppler et al. This is an open-access article distributed under the terms of the Creative Commons Attribution 4.0 International license.

Address correspondence to Heather D. Bean, Heather.D.Bean@asu.edu.

The volatilome of Coccidioides spp. is strongly dependent on its dimorphic life cycle, but volatilomes of $C$. immitis and C. posadasii are similar, indicating that a single suite of biomarkers can be developed for a Valley fever breath test. @Beanchemist

Received 14 January 202

Accepted 22 March 2021

Published 14 April 2021 
United States where the disease is endemic, e.g., Phoenix and Tucson, AZ, and the San Joaquin Valley in California, up to $30 \%$ of community-acquired pneumonias may be caused by Valley fever, and with growing populations in these regions, Valley fever cases in the United States are expected to climb (2). The causative agent of Valley fever is the opportunistic pathogen Coccidioides, a genus of dimorphic fungi comprised of two species, Coccidioides immitis and C. posadasii. In the environment, Coccidioides spp. grow in the soil during rainy periods as saprobic mycelia, which become dormant and form arthroconidia during dry periods. As the soil is disturbed, the arthroconidia can become airborne, and when inhaled, the fungus can initiate a parasitic life cycle as spherules within the lungs of a susceptible host, causing pneumonia. The median duration of human Valley fever pneumonia is 120 days, costing over $\$ 93,000$ per person in direct and indirect costs $(2,3)$. Approximately three-quarters of the diagnosed cases of Valley fever require antifungal therapy (4), and $1 \%$ of those infected will experience disseminated disease, with one-third of those being fatal (4).

A definitive diagnosis of Valley fever requires a positive fungal culture or histological examination of tissue or bodily fluid. However, due to the invasiveness of obtaining suitable lung specimens for culture or histology, serological testing for antibodies against Coccidioides is the most commonly performed diagnostic test. Multiple serological tests are available for $\lg M$, IgG, or complement-fixing antibodies, as is an enzymelinked immunoassay that uses proprietary coccidioidal antigens. However, these tests lack sensitivity for coccidioidomycosis $(5,6)$. Due to the difficulty in diagnosing Valley fever, it is often mistaken for bacterial pneumonia and inappropriately treated with antibiotics (7-10), and the lack of a suitable diagnostic strongly contributes to an unacceptably long 23-day median time to diagnosis (2).

Breath-based diagnostics are a promising novel approach for diagnosing respiratory infections. Numerous in vitro studies have demonstrated that bacterial and fungal respiratory pathogens have unique volatile metabolome (or volatilome) signatures that can be used to differentiate and identify them to the genus, species, and strain levels (11). Thus far, relatively few studies have specifically focused on identifying breath biomarkers for fungal lung diseases. In vitro analyses of the volatiles produced by Aspergillus spp. have shown that fungi produce many compounds that are also frequently detected in bacterial cultures, e.g., the alcohols ethanol and propanol; the ketones acetone, 2-nonanone, 2-undecanone, and 2,3-butanedione; the sulfides dimethyl disulfide and dimethyl trisulfide; and the pyrazines 2-methyl pyrazine and 2,5-dimethyl pyrazine (12-16). However, Aspergillus also produces a wider variety of monoterpenes, sesquiterpenes, and terpenoids, which are less common in bacterial volatilomes $(14,17)$. With this knowledge, Koo et al. used a biologically guided approach to identify volatile biomarkers of pulmonary invasive aspergillosis (IA), focusing on terpenes and terpenoids produced in vitro as putative biomarkers for a breath test (17). They determined that the Aspergillus fumigatus in vitro volatilome contained a distinctive combination of monoterpenes and sesquiterpenes, and in breath, the sesquiterpenes were the most specific for differentiating patients with IA from those with other pulmonary infections (17). This example for $A$. fumigatus establishes a proof of concept that direct detection of fungal metabolites in breath can be used as a noninvasive, species-specific approach to identify the underlying microbial cause of pneumonia.

We are taking an in vitro-guided approach toward developing a Valley fever breath test by first building a catalog of volatile compounds produced by $C$. immitis and $C$. posadasii. In this study, we have cultured six strains of each species and used solidphase microextraction (SPME) and comprehensive two-dimensional gas chromatography-time of flight mass spectrometry (GC $\times$ GC-TOFMS) to characterize the volatile organic compounds (VOCs) produced during mycelial or spherule growth. Although Coccidioides almost exclusively grows as spherules in the host, many in vitro studies on the pathogenesis and diagnosis of coccidioidomycosis have been performed using mycelial cultures, as the saprobic life cycle has historically been easier to cultivate and maintain (18). We expected to find significant differences in the VOCs between species 
TABLE 1 Coccidioides strains used in this study ${ }^{a}$

\begin{tabular}{|c|c|c|c|c|}
\hline Species & Strain & Population $^{b}$ & ATCC catalog no. & NCBI accession no. \\
\hline \multirow[t]{6}{*}{ C. posadasii } & Silveira & $A Z$ & NR-48944 & ABAI00000000.2 \\
\hline & B3221 & $A Z$ & & NA \\
\hline & B3222 & $A Z$ & & NA \\
\hline & RMSCC2343 & TX/MEX/SA & & SRR3468064 \\
\hline & RMSCC3506 & TX/MEX/SA & & SRR3468053 \\
\hline & GT-166 & TX/MEX/SA & & NA \\
\hline \multirow[t]{6}{*}{ C. immitis } & RS & SDMX & NR-48942 & AAEC00000000.3 \\
\hline & RMSCC2395 & SDMX & NR-48938 & NA \\
\hline & RMSCC3505 & SDMX & & NA \\
\hline & RMSCC2006 & SJV & NR-48934 & NA \\
\hline & RMSCC2009 & SJV & & SRR3468015 \\
\hline & RMSCC2010 & SJV & NR-48935 & NA \\
\hline
\end{tabular}

and between life cycles but also posited that there would be a subset of VOCs that are common to Coccidioides spp. during spherule growth, which will serve as our candidate biomarkers for breath test development.

\section{RESULTS}

Coccidioides isolates in this study. The Coccidioides genus is widely distributed throughout North and South America. The genus is composed of two geographically (19) and genetically $(19,20)$ distinct species, C. posadasii and C. immitis, which are further divided into five well-defined populations (21-23). C. posadasii has a more extensive biogeographic distribution and is divided into three populations, occurring in Texas/Venezuela, Mexico/South America, and Arizona. The species C. immitis is divided into two populations found in central and southern California and was recently found in Washington State, representing a newly discovered third distinct population (24, 25). Representative members of all populations cause disease in humans and other mammals (21-23). Due to the magnitude of the observed genetic variation and geographic distribution between species, we hypothesized that metabolomic variation exists. To capture diversity across the volatilome between and within species, we included a total of 12 isolates from both species, representing four populations, all of which were isolated from humans (Table 1).

Characteristics of the Coccidioides volatilome by species and life cycle. We cultured each Coccidioides isolate in biological triplicate using temperatures and oxygen concentrations that induce mycelial or spherule growth but using the same culture medium, facilitating direct comparisons between the VOCs produced during the two life cycles. Using SPME to adsorb VOCs from the headspace of fungal culture filtrates and GC $\times$ GC-TOFMS to analyze the volatilomes, we detected a total of 353 chromatographic peaks that were at least 2-fold more abundant in a Coccidioides culture $(n=72)$ than in the medium controls $(n=6)$ (see Table S1 in the supplemental material). Of the 353 volatiles detected in Coccidioides cultures, 28 were identified at level 1 or 2 and therefore were assigned putative names based on mass spectral and chromatographic data (see "Processing and analysis of chromatographic data" in Materials and Methods, below), which included 13 compounds previously associated with human and environmental fungal pathogens (26) (Table 2). For the unnamed compounds, we assigned chemical classifications to 45 of them, which were assigned a level 3 identification based on a combination of mass spectral and chromatographic characteristics (Table S1).

The total numbers of volatiles produced by C. posadasii and C. immitis were similar, at 291 and 309, respectively (Fig. 1; Table S2). Within each species, the two life cycles 
TABLE 2 Named Coccidioides volatiles and their previous reports in other fungal taxa

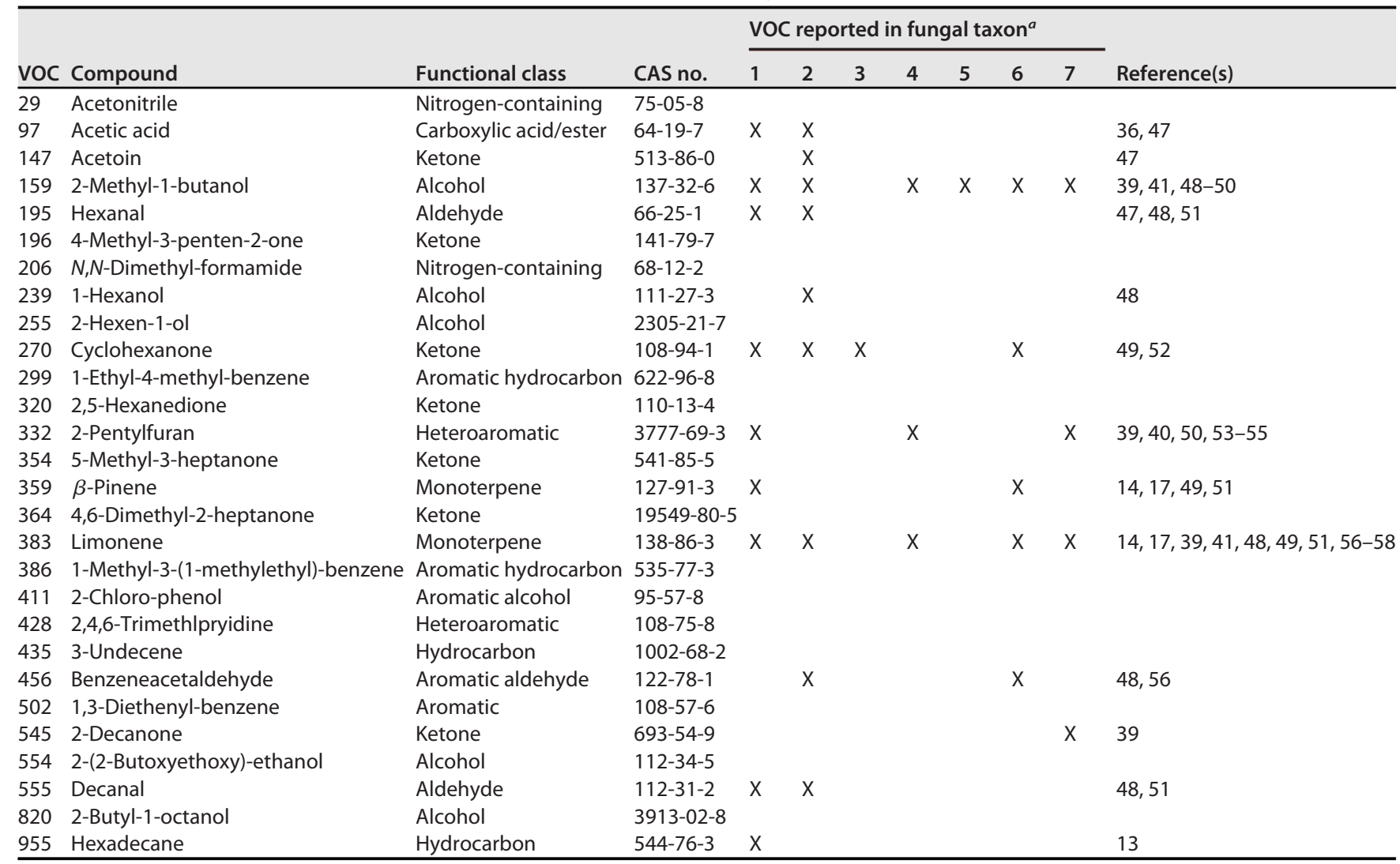

${ }^{a} \mathrm{X}$ indicates that the VOC has been previously detected in the fungal taxa associated with the numbered columns, as follows: 1, Aspergillus spp.; 2, Candida spp.; 3, Cladosporium cladosporioides; 4, Fusarium spp.; 5, Mucor spp.; 6, Penicillium spp.; 7, other species, including environmental fungal species, Trichoderma spp., Stropharia rugosoannulata, and Verticillium longisporum.

are quite divergent in their volatilomes, sharing less than one-third of the total volatilome (Fig. 1A and B). However, within each life cycle, the two species share many similarities, with more than one-half of the VOCs being produced by both $C$. posadasii and C. immitis (Fig. 1C and D). In aggregate across the genus, or subdivided by species, the spherule volatilome is larger than the mycelial volatilome, but this appears to be driven by considerable differences in volatilome sizes observed in a few isolates ( $C$. posadasii B3221 and GT-166 and C. immitis RS) (Table S2).

Analyzing the relative abundances of individual analytes, we observed some statistically significant differences in VOCs produced during the two life cycles but not between the two species. We performed a Mann-Whitney $U$ test using BenjaminiHochberg false discovery rate (FDR) correction comparing all spherule and mycelial VOCs and identified 43 that were significantly different in abundance $(P<0.05)$ (Fig. 2). Of the 43 volatiles, 32 of them had a higher abundance in spherules, compared to 11 in mycelia, which mirrors the overall difference in the sizes of the volatilomes (Fig. 1). Among the VOCs detected in higher abundances in one life cycle than in another were the aromatic hydrocarbon 1-methyl-3-(1-methylethyl)-benzene and the aldehyde hexanal, which are associated with mycelial cultures, while the alcohols 2-(2-butoxyethoxy)ethanol and 2-hexen-1-ol, the ketone cyclohexanone, and the heteroaromatic 2,4,6-trimethylpyridine were associated with spherule cultures. There were no VOCs that were significantly different in relative abundance between C. posadasii and C. immitis.

Most $(>70 \%)$ of the spherule and mycelial VOCs detected in this study were produced by four or fewer Coccidioides strains in each life cycle. Of the 272 spherule volatiles that were detected, only $35(13 \%)$ were detected in at least two-thirds of all spherule cultures (Fig. 3A), 12 of which were also significantly more abundant in spherule 
A

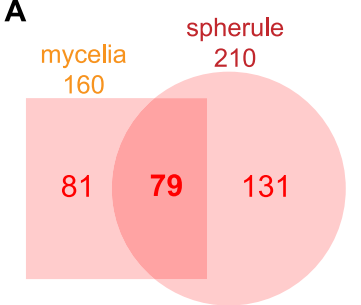

291 C. posadasii VOCs

C

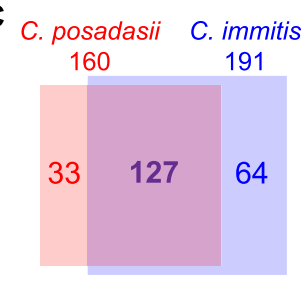

224 mycelia VOCs
B

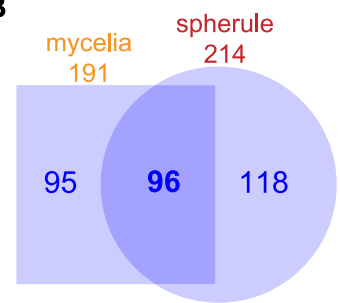

309 C. immitis VOCs

D
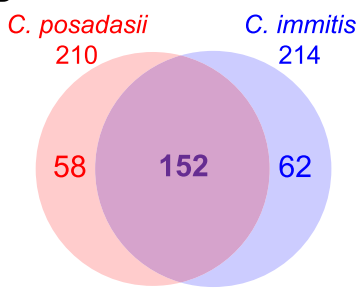

272 spherule VOCs

FIG 1 Numbers of analytes detected in at least a 2-fold-higher abundance in a Coccidioides culture than in the medium controls that are shared and unique among C. posadasii mycelia (square) versus spherules (circle) (A), C. immitis mycelia (square) versus spherules (circle) (B), mycelia of C. posadasii (red) versus C. immitis (blue) (C), and spherules of C. posadasii (red) versus C. immitis (blue) (D).

than in mycelial cultures. Nineteen of 224 mycelial volatiles (8\%) were detected in at least two-thirds of all mycelial samples (Fig. 3B), and only 2 were significantly different between life cycles (VOCs 199 and 428). However, both volatiles were produced in higher relative abundances in spherules. Only two VOCs, VOCs 39 and 748, were detected in two-thirds of both spherule and mycelial samples. Interestingly, the relative abundance of a VOC was usually consistent across all strains. For example, in every spherule culture in which limonene (VOC 383) was detected, it was present at a relative concentration that was between 2- and 10-fold higher than that in the medium (Fig. 3A).

Multivariate analyses of the Coccidioides volatilome. Principal-component analysis (PCA), using the 78 cultures and medium blanks as observations and 353 Coccidioides VOCs as variables, shows that the volatilomes of individual strains and culture conditions are highly reproducible (Fig. S1A) and that they cluster by life cycle and not by species (Fig. 4). This observation is reinforced when we include only mycelia or spherules in the PCA, where no separation between species is observed (Fig. S1B and C), or include only C. immitis or C. posadasii, where we still observe a clear separation by life cycle (Fig. S1D and E). Evaluating these subsets of the data, some interesting variations between strains emerge. The type strain for $C$. immitis is RS; however, in both mycelial and spherule life cycles, we observe that its volatile metabolome is quite unique from those of the other C. immitis strains (Fig. S1). C. posadasii also has a metabolic outlier, RMSCC3506, but only during mycelial growth (Fig. S1B and E). These outliers cannot be fully explained by the size of the volatilomes as being abnormally large or small (Table S2).

Although life cycle and strain differences in the Coccidioides volatilome are observable in the PCA, less than one-quarter of the total variance is captured by the first two principal components. Thus, we also used hierarchical clustering analysis (HCA) to compare the Coccidioides volatilomes (Fig. 5). The HCA reinforces our finding that the life cycle is the most significant contributor to the Coccidioides volatilome, and there are not global similarities in the volatilomes within each species or populations of the species. There are two main clusters of VOCs driving the separation of the mycelial and spherule life cycles in the HCA. Cluster 1 includes 56 VOCs, which are generally present 


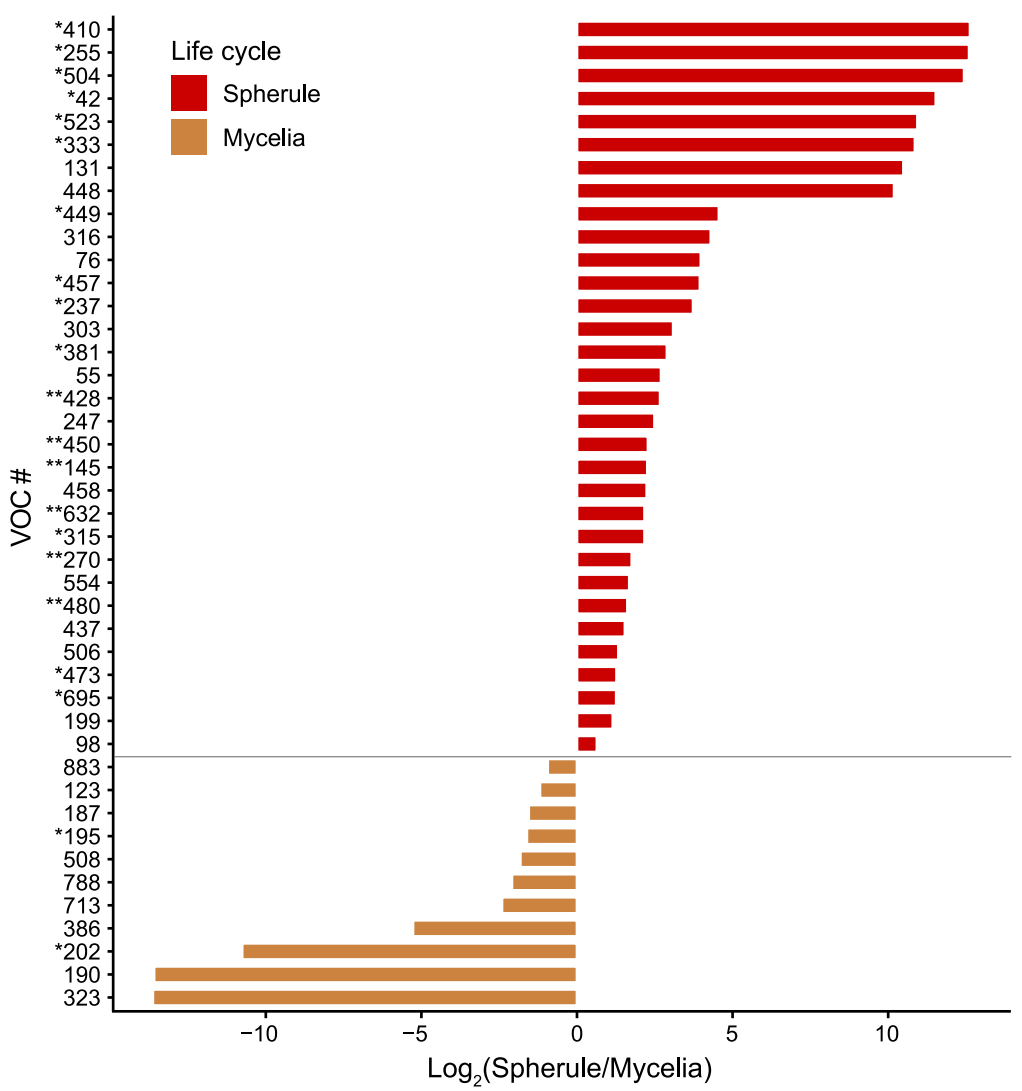

FIG 2 Forty-three volatiles significantly different in abundance between life cycles $(P<0.05)$, expressed as $\log _{2}$ fold changes in peak abundance. Volatiles marked with asterisks indicate greater statistically significant differences $(*, P<0.001 ; * *, P<0.0001)$. Compound identifications: 195 , hexanal; 255, 2-hexen-1-ol; 270, cyclohexanone; 386, 1-methyl-3-(1-methylethyl)-benzene; 428, 2,4,6trimethylpyridine; 554, 2-(2-butoxyethoxy)-ethanol.

in a higher abundance in mycelial samples than in spherule samples, 5 of which are significantly different between life cycles $(P<0.05$ with Benjamini-Hochberg FDR correction) (Table S1). In cluster 1, seven compounds are identified: the hydrocarbon 3undecene, the aromatic hydrocarbons 1-ethyl-4-methyl-benzene and 1-methyl-3-(1methylethyl)-benzene, the alcohol 2-butyl-1-octanol, the aldehyde decanal, and the monoterpenes limonene and $\beta$-pinene. As in the PCA, we find that $C$. immitis RS and $C$. posadasii RMSCC 3506 cluster separately when grown as mycelia, partially driven by the low abundances of VOCs from cluster 1. Cluster 2 includes 16 VOCs that are present in spherule samples while generally being absent or found at a lower abundance in mycelial samples, all of which are significantly different between life cycles $(P<0.05$ with Benjamini-Hochberg FDR correction). Cyclohexanone (a ketone) and 2,4,6-trimethylpyridine (a heteroaromatic) are two VOCs in this cluster that we were able to identify.

\section{DISCUSSION}

The genus Coccidioides is divided into two closely related and putatively allopatric species, with a strong phylogeographic signal and population substructure within each species (20). However, we find that the character of the Coccidioides volatilome is determined by life cycle rather than species. Within each species, the two life cycles have a relatively small overlap in their shared volatilome, with $27 \%$ and $31 \%$ being shared for C. posadasii and C. immitis, respectively (Fig. 1). While there were no volatiles that were significantly different in relative abundance between C. posadasii and C. immitis, we observed 43 
A Strains

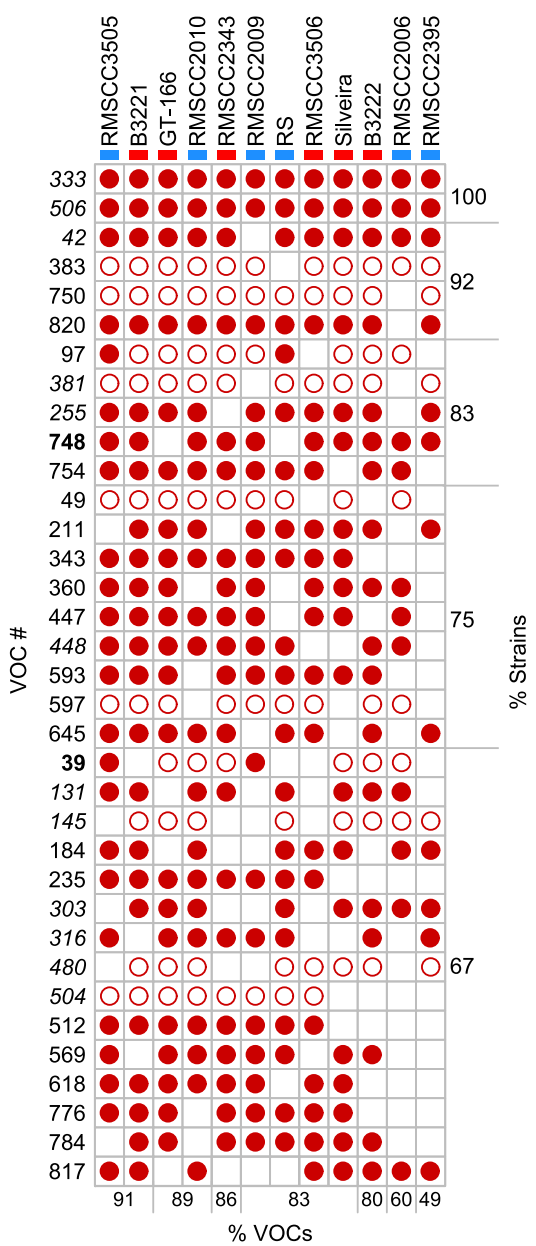

B

Strains

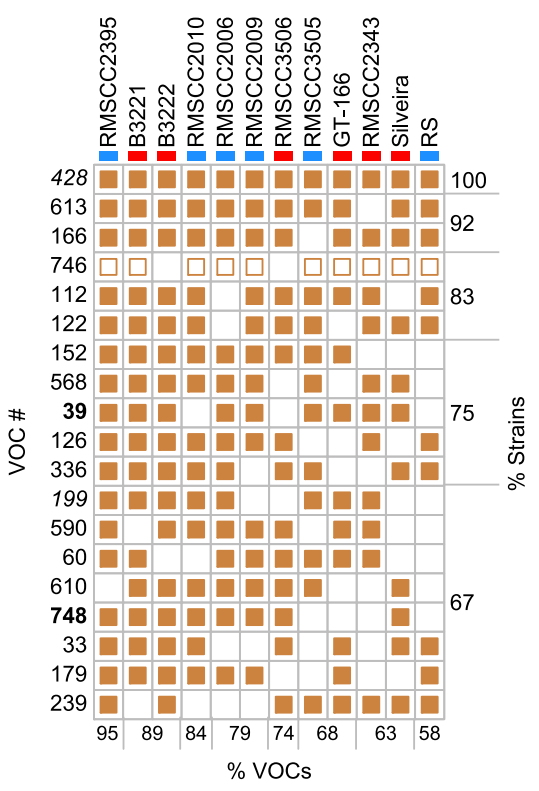

FIG 3 Volatiles detected in at least two-thirds of all Coccidioides strains in spherule (A) or mycelial (B) cultures, with VOCs in boldface type detected in both life cycles and those in italic type detected in statistically significantly different abundances between life cycles $(P<0.05)$. Open shapes indicate VOCs with at least a 2 -fold-higher relative abundance than in medium blanks, and solid shapes indicate VOCs with at least a 10-fold-higher relative abundance. Strains are color-coded as blue for $C$. immitis and red for $C$. posadasii. The percentages of listed VOCs detected in each strain and the percentages of strains producing each VOC are noted at the bottom and right sides of each chart, respectively. Compound identifications: 97, acetic acid; 239, 1-hexanol; 255, 2-hexen-1-ol; 383, limonene; 428, 2,4,6,-trimethylpyridine; 820, 2-butyl-1-octanol.

statistically significant differences in the VOCs produced during the two life cycles (Fig. 2). This global difference between life cycles is the primary driver of separation that we observed in multivariate analyses using PCA (Fig. 4) and HCA (Fig. 5). These findings are in line with the large-scale transcriptional changes that occur when Coccidioides species transitions from mycelial to spherule growth: between $20 \%$ and $40 \%$ of the Coccidioides genes are differentially regulated during the shift between the environmental and parasitic life cycles (27-30). It has also been shown that the proteomes of the two life cycles are distinct, with statistically significant differences between mycelial and spherule life cycles (31). In contrast to the significant differences in the volatilomes between life cycles, we observed no uniform differences between the volatilomes of $C$. posadasii and C. immitis. There is a high degree of overlap (56 to $57 \%$ ) between the species' volatilomes when comparing the same life cycle (Fig. 1). This similarity is also visible in the PCA (Fig. 4; see also Fig. S1 in the supplemental material) and HCA (Fig. 5), as the volatilomes do not cluster by species even when we 


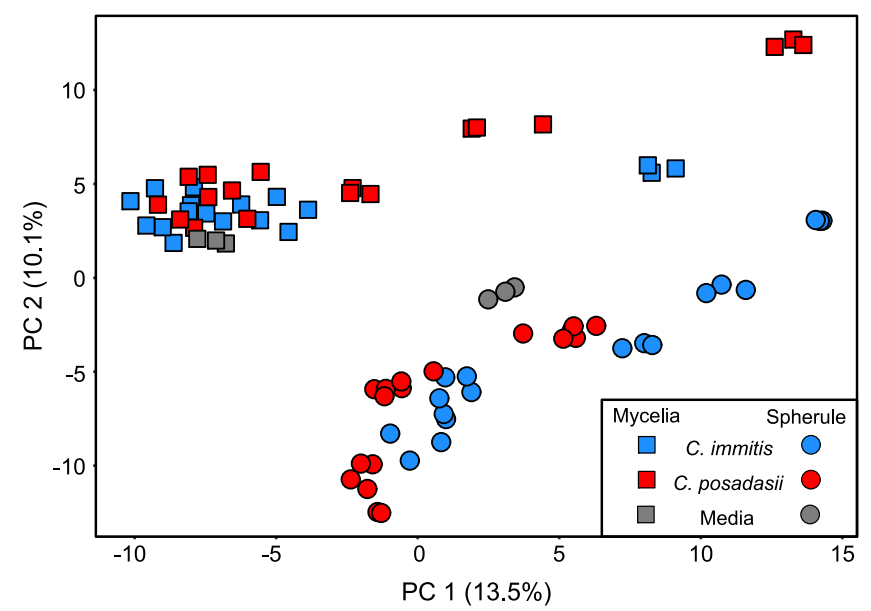

FIG 4 Principal-component analysis (PCA) score plot using 353 VOCs as features, produced by C. immitis (blue) and C. posadasii (red) when cultured under conditions that induced mycelial (squares) or spherule (circles) morphologies. Blank media are shown in gray. Fungal cultures and medium blanks were analyzed in triplicate, yielding 72 fungal and 6 blank observations. PCA score plots with the observations colored by strain are shown in Fig. S1 in the supplemental material.

control for the life cycle. Global similarities between C. posadasii and C. immitis have also been demonstrated at the protein level $(31,32)$.

The lack of significant differences between the C. posadasii and C. immitis volatilomes is due, in part, to large differences observed between strains of the same species; the volatilome highlights some unique aspects of a few of the individual Coccidioides strains that we analyzed. For example, RS is the type strain of C. immitis; however, it appears to be a metabolic outlier during both life cycles (Fig. S1) but especially during mycelial growth (Fig. 5). Additionally, the mycelial life cycle of C. posadasii RMSCC3506 appears to be a metabolic outlier compared to the other strains grown as mycelia (Fig. 5; Fig. S1). These mycelial outliers appear to be driven, at least in part, by the lack of volatiles that make up cluster 1 in the HCA (Fig. 5). None of the 56 VOCs found in cluster 1 , which are more abundant in mycelial than in spherule cultures, are detected in RMSCC3506 cultures, and only 2 are detected in RS mycelial cultures. These two strains also lack VOCs 39 and 748, which were detected in the majority of strains for both life cycles but absent from both life cycles of RS and mycelial cultures of RMSCC3506 (Fig. 3). Additionally, none of the 11 VOCs that were significantly more abundant in mycelial than in spherule cultures (Fig. 2 and Table S1) are detected in RMSCC3506, and only 2 are detected in RS. Beyond contributing to high levels of volatilome variance in Coccidioides, the significance of the strain-level differences relative to fungal biology or infection is not known. Variation in virulence among strains in murine models of coccidioidomycosis has been observed but has not been tested in the context of species or with a mechanistic hypothesis (33). In future studies of mouse models and human infections, we plan to investigate correlations between the Coccidioides volatilome and virulence and infection outcomes.

It should be noted that although the spherule and mycelial media had the same composition, we detected different VOCs in the headspace of the blanks (Fig. 4 and 5; Table S1). We interpret these differences as being the influence of the cultures on the blanks rather than the influence of the blank media on the cultures. Because the medium blanks were incubated and processed in parallel with their respective samples, we posit that the blank media absorbed volatiles from the culture environment (e.g., from fungal spherule or mycelial cultures in shared incubators), which introduced differences in their volatilomes. An analysis of fresh blank media (not incubated with the 


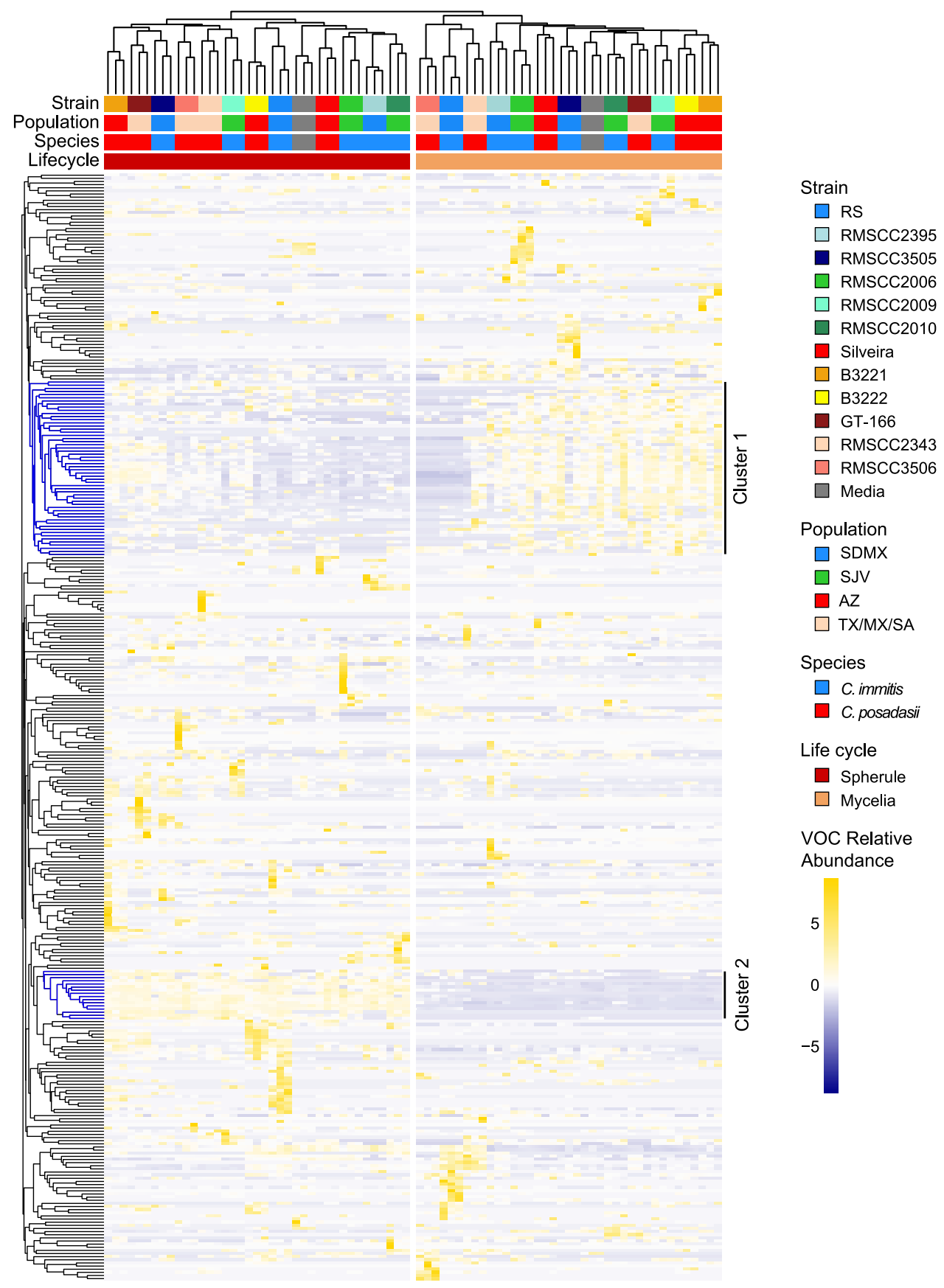

FIG 5 Hierarchical clustering analysis (HCA) of 72 fungal cultures and 6 medium blanks (columns) based on the relative abundances of 353 volatiles (rows). Clustering of fungal and blank samples was performed using Pearson correlations, and clustering of volatiles was performed using Euclidean distance, both with average linkages. Samples are color-coded by strain, population, species, and life cycle, as labeled in the key; medium blanks are in gray.

cultures) would be required to test this hypothesis. Our data indicate that the spherule media absorbed a higher abundance of compounds than the mycelial media (Table S1), which may be due to the spherule life cycle producing higher numbers and/or abundances of individual compounds. Therefore, it is possible that there are additional significant spherule volatiles that were not identified in this study since a VOC was defined as "detected" based on its relative abundance versus medium blanks. 
Future in vitro work needs to be mindful of volatile absorption by the cultures and blanks within a shared space such as an incubator.

The results from this study highlight two crucial factors that need to be considered in the development and translation of volatilomics (and other functional genomics data) into biomarkers. First, there can be a significant amount of intraspecies variation such that the volatilome of a single strain is not representative of the species as a whole $(17,34-36)$. Therefore, an analysis of multiple strains should be performed to identify a core volatilome, and the more genetically and phenotypically diverse the species, the more strains will need to be analyzed to determine the consensus set of volatiles produced by the majority of them. Second, our data show that the growth phase of the organism has a strong influence on the volatilome, and therefore, using in vitro culture conditions that can most closely replicate the in vivo environment and microbial growth phases may increase the likelihood of putative biomarker translation from bench to bedside. In addition to controlling the growth phase of dimorphic organisms, macronutrient, micronutrient, and oxygen availability should all be considered in the development of in vitro model systems that replicate the in vivo physiology of the pathogen (14, 17, 36-41). In this study, we observed 35 VOCs present in at least $67 \%$ of all spherule strains (Fig. 3); as this is the parasitic life cycle found within the host, we hypothesize that the most highly conserved spherule VOCs have the highest probability of translating to animal models and to human Valley fever breath biomarkers.

The first priority for diagnosing pneumonia etiology is determining whether the underlying cause is bacterial, fungal, or viral, as this will guide the selection of antibiotics, antifungals, or neither for treating the infection. We have initiated a study to directly compare coccidioidomycosis, bacterial pneumonia, and viral pneumonia in Arizona residents to determine the specificity of the Coccidioides spherule volatiles for diagnosing the fungal pneumonia that is endemic in this region of the United States. There have not yet been any human breath studies designed to compare multiple fungal and bacterial pneumonias to determine if there might also be a universal set of fungal breath volatiles, but given the limited overlap in the regions of the United States where fungal pneumonias are endemic (42), a set of universal fungal breath volatiles will not be required to substantially improve pneumonia diagnostics. It may be of clinical or epidemiological interest to differentiate coccidioidomycosis from other fungal pneumonias such as aspergillosis, blastomycosis, histoplasmosis, or cryptococcosis. Previous studies have shown that bacterial lung pathogens have unique and reproducible volatilomes that can be used to detect and identify organisms at the genus, species, or strain level (11), and therefore, differentiating fungal pneumonias is feasible.

Conclusion. The Coccidioides volatilome is strongly influenced by the saprobic versus parasitic life cycles, while the two species, C. posadasii and C. immitis, are indistinguishable by their volatile profiles, in part due to high strain-to-strain variability within the species. We identified 35 VOCs produced by the majority of the Coccidioides strains when grown as spherules, the parasitic form of the fungus found in Valley fever lung infections and in disseminated fungal disease. Our results show that as future efforts are made to develop biomarkers for Coccidioides and other mycoses caused by dimorphic fungi, it is vitally important to control for the fungal life cycle to represent parasitic growth in the host and to sample multiple strains of the pathogen to identify volatiles that are more highly conserved within the infectious genus or species.

\section{MATERIALS AND METHODS}

Fungal isolates and growth conditions. The Coccidioides isolates used in the study were obtained from human patients and are listed in Table 1. All Coccidioides isolates were grown under biosafety level 3 (BSL-3) containment, under conditions that induce mycelial or spherule growth.

Fungal growth conditions were performed as described previously $(18,43)$ and are summarized here in brief. For mycelial growth, a 50-ml vented falcon tube containing $10 \mathrm{ml}$ of RPMI medium (filter-sterilized RPMI $1640,10 \%$ fetal bovine serum) was inoculated with a $1-\mathrm{cm}$ by $1-\mathrm{cm} 2 \times$ glucose yeast extract (GYE) agar plug for each strain. These plates were inoculated using $100 \mu \mathrm{l}$ of a glycerol stock, spread across the plate, and cultured at $30^{\circ} \mathrm{C}$ for 2 weeks. Control RPMI medium was inoculated with a plug from sterile $2 \times$ GYE agar medium. Each sample, including the medium control, was prepared in triplicate. Cultures were grown on a shaking incubator at $150 \mathrm{rpm}$ at $30^{\circ} \mathrm{C}$ for $96 \mathrm{~h}$. For spherule cultures, a 
50- $\mathrm{ml}$ vented falcon tube containing $10 \mathrm{ml}$ of RPMI medium was inoculated to a final concentration of $1.0 \times 10^{5}$ arthroconidia/ml in $1 \times$ phosphate-buffered saline (PBS). Arthroconidia were grown and harvested as previously described $(18,43)$. Strains RMSCC2343 and RMSCC3505 did not produce enough conidia to achieve $1.0 \times 10^{5}$ arthroconidia/ml and were inoculated at $7.0 \times 10^{4}$ and $4.0 \times 10^{4}$ arthroconi$\mathrm{dia} / \mathrm{ml}$, respectively. Control medium was inoculated with $1 \mathrm{ml}$ of sterile $1 \times \mathrm{PBS}$. Cultures were grown on a shaking incubator at $150 \mathrm{rpm}$ at $39^{\circ} \mathrm{C}$ in $10 \% \mathrm{CO}_{2}$ for $96 \mathrm{~h}$. Mycelial and spherule cultures were spun at $12,000 \times g$ at $4^{\circ} \mathrm{C}$ for $10 \mathrm{~min}$ to pellet the cells. The supernatant was removed, placed in a Nanosep MF centrifugal device with a Bio-Inert membrane $0.2-\mu \mathrm{m}$ spin filter, and centrifuged at $3,200 \times g$ for $4 \mathrm{~min}$. The filtrate was stored at $-80^{\circ} \mathrm{C}$ until volatile metabolomics analysis.

To ensure the sterility of the filtrates for metabolomics analyses outside a BSL-3 containment facility, $10 \%$ of all sample filtrates were plated on $2 \times \mathrm{GYE}$ and incubated at $30^{\circ} \mathrm{C}$ for $96 \mathrm{~h}$ to ensure the complete removal of viable pathogen particles. No growth was observed in any replicate.

Volatile metabolomics analysis by SPME-GCXGC-TOFMS. The Coccidioides species culture filtrates and medium blanks were allowed to thaw at $4^{\circ} \mathrm{C}$ overnight, and $2 \mathrm{ml}$ was then transferred and sealed in sterilized 10-ml GC headspace vials with polytetrafluoroethylene (PTFE)-silicone septum screw caps. All samples were stored for up to 12 days at $4^{\circ} \mathrm{C}$ until analyzed. Samples were randomized for analysis. Volatile metabolite sampling was performed by solid-phase microextraction (SPME) using a Gerstel multipurpose sampler directed by Maestro software. Sample extraction and injection parameters are provided in Table S3 in the supplemental material (autosampler method). Volatile metabolite analysis was performed by two-dimensional gas chromatography-time of flight mass spectrometry (GC $\times$ GCTOFMS) using Leco (St. Joseph, MI) Pegasus 4D and Agilent 7890 GC instruments. Chromatographic, mass spectrometric, and peak detection parameters are provided in Table $\mathrm{S} 3(\mathrm{GC} \times \mathrm{GC}$ method and mass spectrometry method). An external alkane standard mixture $\left(C_{8}\right.$ to $C_{20}$; Sigma-Aldrich, St. Louis, $\left.M O\right)$ was sampled multiple times for calculating retention indices (RIs). The injection, chromatographic, and mass spectrometric methods for analyzing the alkane standards were the same as the methods for the samples.

Processing and analysis of chromatographic data. Data collection, processing, and alignment were performed using ChromaTOF software version 4.71 with the Statistical Compare package (Leco Corp.), using the parameters listed in Table S3 (data processing method).

Peaks were assigned a putative identification based on mass spectral similarity and RI data, and the confidence of those identifications is indicated by assigning levels 1 to 4 (with 1 being the highest) (44). Peaks with a level 1 identification were identified based on mass spectral and RI matches with external standards. Peaks with a level 2 identification were identified based on $\geq 800$ mass spectral matches by a forward search of the NIST 2011 library and RIs that are consistent with the midpolar Rxi-624Sil stationary phase, as previously described (34), but using an RI range of 0 to $43 \%$ (empirically determined by comparing the Rxi-624Sil Rls for Grob mix standards to published polar and nonpolar values). Level 1, 2, and 3 compounds were assigned to chemical functional groups based upon characteristic mass spectral fragmentation patterns and second-dimension retention times, as previously described (35). Level 4 compounds have mass spectral matches of $<600$ or Rls that do not match previously published values and are reported as unknowns.

Data postprocessing and statistical analyses. The data postprocessing steps are depicted in Fig. S2. Before statistical analyses, compounds eluting prior to $358 \mathrm{~s}$ (acetone retention time) and siloxanes (i.e., chromatographic artifacts) were removed from the peak table. Peaks that were present in only one of the three biological replicates were imputed to zero for that sample, while missing values for peaks that were present in two out of three biological replicates were imputed to half of the minimum value across all biological replicates. The relative abundances of compounds across chromatograms were normalized using probabilistic quotient normalization (PQN) (45) in R version 3.4.3. The data were $\log _{10}$ transformed, intraclass correlation coefficients (ICCs) were calculated using R ICC package version 2.3.0, and peaks with an ICC of $<0.75$ were not further processed. Analytes were retained for further analysis if they were at least 2-fold more abundant in any Coccidioides culture than in the medium controls and were considered to have been detected in that culture. Principal-component analysis was performed using R factoextra package version 1.0 .5 with the biological replicates as observations and the absolute peak intensities (mean centered and scaled to unit variance) as variables. The relatedness of samples based on their volatile metabolomes was assessed using hierarchical clustering analysis on Pearson's correlation between isolates and the Euclidean distance between volatiles using R pheatmap package version 1.0.12. Geometric means of the biological replicates were calculated and used to determine the statistical difference in abundances between life cycles or species using a Mann-Whitney $\mathrm{U}$ test and Benjamini-Hochberg false discovery rate correction with an $\alpha$ value of 0.05 , using $\mathrm{R}$ stats package version 3.5.3. The relative abundance of volatiles significantly different between life cycles was calculated by dividing the mean mycelial peak abundance by the mean spherule peak abundance.

Data availability. Metabolomic data (chemical feature peak areas and retention time information) included in this study are available at the NIH Common Fund's National Metabolomics Data Repository (NMDR) website, the Metabolomics Workbench, at www.metabolomicsworkbench.org, where it has been assigned project identifier PR0001064 and study identifier ST001659 (https://doi.org/10.21228/ M85H6W).

\section{SUPPLEMENTAL MATERIAL}

Supplemental material is available online only.

FIG S1, JPG file, 0.5 MB. 
FIG S2, JPG file, $0.3 \mathrm{MB}$.

TABLE S1, XLSX file, 0.1 MB.

TABLE S2, PDF file, $0.1 \mathrm{MB}$.

TABLE S3, PDF file, $0.2 \mathrm{MB}$.

\section{ACKNOWLEDGMENTS}

Funding for this work was provided by Arizona Biomedical Research Centre award ADHS18-198861 (H.D.B.). Supplemental funding for publication was provided by an Arizona State University Graduate and Professional Student Association publication fee grant awarded to E.A.H.K.

We thank John Galgiani and George Thompson, who provided Coccidioides clinical isolates to B.M.B.

We declare that the research was conducted in the absence of any commercial or financial relationships that could be construed as a potential conflict of interest.

\section{REFERENCES}

1. Chiller T. 2019. Overview of endemic mycoses. NIAID, Rockville, MD.

2. Nguyen C, Barker BM, Hoover S, Nix DE, Ampel NM, Frelinger JA, Orbach MJ, Galgiani JN. 2013. Recent advances in our understanding of the environmental, epidemiological, immunological, and clinical dimensions of coccidioidomycosis. Clin Microbiol Rev 26:505-525. https://doi.org/10 .1128/CMR.00005-13.

3. Wilson $L$, Ting J, Lin $H$, Shah $R$, MacLean M, Peterson MW, Stockamp $N$, Libke R, Brown P. 2019. The rise of Valley fever: prevalence and cost burden of coccidioidomycosis infection in California. Int J Environ Res Public Health 16:1113. https://doi.org/10.3390/ijerph16071113.

4. Odio CD, Marciano BE, Galgiani JN, Holland SM. 2017. Risk factors for disseminated coccidioidomycosis, United States. Emerg Infect Dis 23:308-311. https://doi.org/10.3201/eid2302.160505.

5. Grys TE, Brighton A, Chang Y-H, Liesman R, Bolster LaSalle C, Blair JE. 2019. Comparison of two FDA-cleared ElA assays for the detection of Coccidioides antibodies against a composite clinical standard. Med Mycol 57:595-600. https://doi.org/10.1093/mmy/myy094.

6. Kassis C, Durkin M, Holbrook E, Myers R, Wheat L. 2021. Advances in diagnosis of progressive pulmonary and disseminated coccidioidomycosis. Clin Infect Dis 72:968-975. https://doi.org/10.1093/cid/ciaa188.

7. Singh VR, Smith DK, Lawerence J, Kelly PC, Thomas AR, Spitz B, Sarosi GA 1996. Coccidioidomycosis in patients infected with human immunodeficiency virus: review of 91 cases at a single institution. Clin Infect Dis 23:563-568. https://doi.org/10.1093/clinids/23.3.563.

8. Muñoz-Hernández B, Palma-Cortés G, Cabello-Gutiérrez C, MartínezRivera MA. 2014. Parasitic polymorphism of Coccidioides spp. BMC Infect Dis 14:213. https://doi.org/10.1186/1471-2334-14-213.

9. Ampel NM. 2010. The diagnosis of coccidioidomycosis. F1000 Med Rep 2:2. https://doi.org/10.3410/M2-2.

10. Galgiani JN, Grace GM, Lundergan LL. 1991. New serologic tests for early detection of coccidioidomycosis. J Infect Dis 163:671-674. https://doi .org/10.1093/infdis/163.3.671

11. Davis CE, Hill JE, Frank M, McCartney MM, Schivo M, Bean HD. 2020. Breath analysis for respiratory infections, p 335-347. In Beauchamp JD, Davis CE, Pleil JD (ed), Breathborne biomarkers and the human volatilome, 2nd ed. Elsevier, Amsterdam, Netherlands.

12. Chippendale TWE, Gilchrist FJ, Španěl P, Alcock A, Lenney W, Smith D 2014. Quantification by SIFT-MS of volatile compounds emitted by in vitro cultures of S. aureus, S. pneumoniae and $H$. influenzae isolated from patients with respiratory diseases. Anal Methods 6:2460-2472. https://do .org/10.1039/c4ay00209a.

13. Neerincx AH, Geurts BP, Habets MFJ, Booij JA, van Loon J, Jansen JJ, Buydens LMC, van Ingen J, Mouton JW, Harren FJM, Wevers RA, Merkus PJFM, Cristescu SM, Kluijtmans LAJ. 2016. Identification of Pseudomonas aeruginosa and Aspergillus fumigatus mono- and co-cultures based on volatile biomarker combinations. J Breath Res 10:016002. https://doi.org/ 10.1088/1752-7155/10/1/016002.

14. Heddergott C, Calvo AM, Latge JP. 2014. The volatome of Aspergillus fumigatus. Eukaryot Cell 13:1014-1025. https://doi.org/10.1128/EC.00074-14.

15. Rees CA, Stefanuto P-H, Beattie SR, Bultman KM, Cramer RA, Hill JE. 2017. Sniffing out the hypoxia volatile metabolic signature of
Aspergillus fumigatus. J Breath Res 11:036003. https://doi.org/10.1088/ 1752-7163/aa7b3e.

16. Dickschat JS. 2017. Fungal volatiles-a survey from edible mushrooms to moulds. Nat Prod Rep 34:310-328. https://doi.org/10.1039/c7np00003k.

17. Koo S, Thomas HR, Daniels SD, Lynch RC, Fortier SM, Shea MM, Rearden P, Comolli JC, Baden LR, Marty FM. 2014. A breath fungal secondary metabolite signature to diagnose invasive aspergillosis. Clin Infect Dis 59:1733-1740. https://doi.org/10.1093/cid/ciu725.

18. Mead HL, Van Dyke MCC, Barker BM. 2020. Proper care and feeding of Coccidioides: a laboratorian's guide to cultivating the dimorphic stages of C. immitis and C. posadasii. Curr Protoc Microbiol 58:e113. https://doi.org/ 10.1002/cpmc.113.

19. Fisher MC, Koenig GL, White TJ, San-Blas G, Negroni R, Alvarez IG, Wanke B, Taylor JW. 2001. Biogeographic range expansion into South America by Coccidioides immitis mirrors New World patterns of human migration. Proc Natl Acad Sci U S A 98:4558-4562. https://doi.org/10.1073/pnas .071406098 .

20. Fisher MC, Koenig GL, White TJ, Taylor JW. 2002. Molecular and phenotypic description of Coccidioides posadasii sp. nov., previously recognized as the non-California population of Coccidioides immitis. Mycologia 94:73-84. https://doi.org/10.1080/15572536.2003.11833250.

21. Engelthaler DM, Roe CC, Hepp CM, Teixeira M, Driebe EM, Schupp JM, Gade L, Waddell V, Komatsu K, Arathoon E, Logemann H, Thompson GR, III, Chiller T, Barker B, Keim P, Litvintseva AP. 2016. Local population structure and patterns of Western Hemisphere dispersal for Coccidioides spp., the fungal cause of Valley fever. mBio 7:e00550-16. https://doi.org/10 $.1128 / \mathrm{mBio} .00550-16$.

22. Teixeira MM, Barker BM. 2016. Use of population genetics to assess the ecology, evolution, and population structure of Coccidioides. Emerg Infect Dis 22:1022-1030. https://doi.org/10.3201/eid2206.151565.

23. Teixeira MM, Alvarado P, Roe CC, Thompson GR, III, Patané JSL, Sahl JW, Keim P, Galgiani JN, Litvintseva AP, Matute DR, Barker BM. 2019. Population structure and genetic diversity among isolates of Coccidioides posadasii in Venezuela and surrounding regions. mBio 10:e01976-19. https:// doi.org/10.1128/mBio.01976-19.

24. Marsden-Haug N, Hill H, Litvintseva AP, Engelthaler DM, Driebe EM, Roe CC, Ralston C, Hurst S, Goldoft M, Gade L, Wohrle R, Thompson GR, III, Brandt ME, Chiller T, Centers for Disease Control and Prevention. 2014. Coccidioides immitis identified in soil outside of its known range-Washington, 2013. MMWR Morb Mortal Wkly Rep 63:450.

25. Litvintseva AP, Marsden-Haug N, Hurst S, Hill H, Gade L, Driebe EM, Ralston C, Roe C, Barker BM, Goldoft M, Keim P, Wohrle R, Thompson GR, III, Engelthaler DM, Brandt ME, Chiller T. 2015. Valley fever: finding new places for an old disease. Coccidioides immitis found in Washington state soil associated with recent human infection. Clin Infect Dis 60:e1-e3. https://doi.org/10.1093/cid/ciu681.

26. Hérivaux A, Gonçalves SM, Carvalho A, Cunha C. 2020. Microbiota-derived metabolites as diagnostic markers for respiratory fungal infections. J Pharm Biomed Anal 189:113473. https://doi.org/10.1016/j.jpba.2020 .113473 . 
27. Mead HL, Roe CC, Higgins Keppler EA, Caballero Van Dyke MC, Laux KL, Funke AL, Miller KJ, Bean HD, Sahl JW, Barker BM. 2020. Defining critical genes during spherule remodeling and endospore development in the fungal pathogen, Coccidioides posadasii. Front Genet 11:483. https://doi .org/10.3389/fgene.2020.00483.

28. Caballero Van Dyke MC, Teixeira MM, Barker BM. 2019. Fantastic yeasts and where to find them: the hidden diversity of dimorphic fungal pathogens. Curr Opin Microbiol 52:55-63. https://doi.org/10.1016/j.mib.2019 .05.002.

29. Viriyakosol S, Singhania A, Fierer J, Goldberg J, Kirkland TN, Woelk CH 2013. Gene expression in human fungal pathogen Coccidioides immitis changes as arthroconidia differentiate into spherules and mature. BMC Microbiol 13:121. https://doi.org/10.1186/1471-2180-13-121.

30. Whiston E, Wise HZ, Sharpton TJ, Jui G, Cole GT, Taylor JW. 2012. Comparative transcriptomics of the saprobic and parasitic growth phases in Coccidioides spp. PLoS One 7:e41034. https://doi.org/10.1371/journal.pone .0041034 .

31. Mitchell NM, Grys TE, Lake DF. 2020. Carbo-loading in Coccidioides spp.: a quantitative analysis of CAZyme abundance and resulting glycan popula tions. Glycobiology 30:186-196. https://doi.org/10.1093/glycob/cwz092.

32. Mitchell NM, Sherrard AL, Dasari S, Magee DM, Grys TE, Lake DF. 2018. Proteogenomic re-annotation of Coccidioides posadasii strain Silveira. Proteomics 18:1700173. https://doi.org/10.1002/pmic.201700173.

33. Friedman L, Smith CE, Roessler WG, Berman EJ. 1956. The virulence and infectivity of twenty-seven strains of Coccidioides immitis. Am J Hyg 64:198-210. https://doi.org/10.1093/oxfordjournals.aje.a119834.

34. Bean HD, Rees CA, Hill JE. 2016. Comparative analysis of the volatile metabolomes of Pseudomonas aeruginosa clinical isolates. J Breath Res 10:047102. https://doi.org/10.1088/1752-7155/10/4/047102.

35. Davis TJ, Karanjia AV, Bhebhe CN, West SB, Richardson M, Bean HD. 2020. Pseudomonas aeruginosa volatilome characteristics and adaptations in chronic cystic fibrosis lung infections. mSphere 5:e00843-20. https://do .org/10.1128/mSphere.00843-20.

36. Bazemore RA, Feng J, Cseke L, Podila GK. 2012. Biomedically important pathogenic fungi detection with volatile biomarkers. J Breath Res 6:016002. https://doi.org/10.1088/1752-7155/6/1/016002.

37. Koehler T, Ackermann I, Brecht D, Uteschil F, Wingender J, Telgheder U, Schmitz OJ. 2020. Analysis of volatile metabolites from in vitro biofilms of Pseudomonas aeruginosa with thin-film microextraction by thermal desorption gas chromatography-mass spectrometry. Anal Bioanal Chem 412:2881-2892. https://doi.org/10.1007/s00216-020-02529-4.

38. Jenkins CL, Bean HD. 2020. Dependence of the staphylococcal volatilome composition on microbial nutrition. Metabolites 10:347. https://doi.org/ 10.3390/metabo10090347.

39. Nemčovič M, Jakubíková L, Víden I, Farkaš V. 2008. Induction of conidiation by endogenous volatile compounds in Trichoderma spp. FEMS Microbiol Lett 284:231-236. https://doi.org/10.1111/j.1574-6968.2008.01202.x.

40. Gao P, Korley F, Martin J, Chen BT. 2002. Determination of unique microbial volatile organic compounds produced by five Aspergillus species commonly found in problem buildings. AlHA J (Fairfax, Va) 63:135-140. https://doi.org/10.1080/15428110208984696.

41. Fiedler K, Schütz E, Geh S. 2001. Detection of microbial volatile organic compounds (MVOCs) produced by moulds on various materials. Int J Hyg Environ Health 204:111-121. https://doi.org/10.1078/1438-4639-00094.

42. Ashraf N, Kubat RC, Poplin V, Adenis AA, Denning DW, Wright L, McCotter O, Schwartz IS, Jackson BR, Chiller T, Bahr NC. 2020. Re-drawing the maps for endemic mycoses. Mycopathologia 185:843-865. https://doi.org/10 .1007/s11046-020-00431-2.

43. Mead HL, Teixeira MDM, Galgiani JN, Barker BM. 2019. Characterizing in vitro spherule morphogenesis of multiple strains of both species of Coccidioides. Med Mycol 57:478-488. https://doi.org/10.1093/mmy/myy049.
44. Sumner LW, Amberg A, Barrett D, Beale MH, Beger R, Daykin CA, Fan TW-M, Fiehn O, Goodacre R, Griffin JL, Hankemeier T, Hardy N, Harnly J, Higashi R, Kopka J, Lane AN, Lindon JC, Marriott P, Nicholls AW, Reily MD, Thaden JJ, Viant MR. 2007. Proposed minimum reporting standards for chemical analysis Chemical Analysis Working Group (CAWG) Metabolomics Standards Initiative (MSI). Metabolomics 3:211-221. https://doi.org/10.1007/s11306-007 $-0082-2$.

45. Dieterle F, Ross A, Schlotterbeck G, Senn H. 2006. Probabilistic quotien normalization as robust method to account for dilution of complex biological mixtures. Application in ${ }^{1} \mathrm{H}$ NMR metabonomics. Anal Chem 78:4281-4290. https://doi.org/10.1021/ac051632c.

46. Barker BM, Tabor JA, Shubitz LF, Perrill R, Orbach MJ. 2012. Detection and phylogenetic analysis of Coccidioides posadasii in Arizona soil samples. Fungal Ecol 5:163-176. https://doi.org/10.1016/j.funeco.2011.07.010.

47. Filipiak W, Beer R, Sponring A, Filipiak A, Ager C, Schiefecker A, Lanthaler S, Helbok R, Nagl M, Troppmair J, Amann A. 2015. Breath analysis for in vivo detection of pathogens related to ventilator-associated pneumonia in intensive care patients: a prospective pilot study. J Breath Res 9:016004. https://doi.org/10.1088/1752-7155/9/1/016004.

48. Costa CP, Bezerra AR, Almeida A, Rocha SM. 2020. Candida species (volatile) metabotyping through advanced comprehensive two-dimensional gas chromatography. Microorganisms 8:1911. https://doi.org/10.3390/ microorganisms8121911.

49. Matysik S, Herbarth O, Mueller A. 2009. Determination of microbial volatile organic compounds (MVOCs) by passive sampling onto charcoal sorbents. Chemosphere 76:114-119. https://doi.org/10.1016/j.chemosphere .2009.02.010.

50. Müller A, Faubert $P$, Hagen $M$, zu Castell W, Polle A, Schnitzler J-P, Rosenkranz M. 2013. Volatile profiles of fungi-chemotyping of species and ecological functions. Fungal Genet Biol 54:25-33. https://doi.org/10 .1016/j.fgb.2013.02.005.

51. Polizzi V, Adams A, Malysheva SV, De Saeger S, Van Peteghem C, Moretti A, Picco AM, De Kimpe N. 2012. Identification of volatile markers for indoor fungal growth and chemotaxonomic classification of Aspergillus species. Fungal Biol 116:941-953. https://doi.org/10.1016/j.funbio.2012 .06 .001 .

52. Perl T, Jünger M, Vautz W, Nolte J, Kuhns M, Borg-von Zepelin M, Quintel M. 2011. Detection of characteristic metabolites of Aspergillus fumigatus and Candida species using ion mobility spectrometry-metabolic profiling by volatile organic compounds. Mycoses 54:e828-e837. https://doi .org/10.1111/j.1439-0507.2011.02037.x.

53. Syhre M, Scotter JM, Chambers ST. 2008. Investigation into the production of 2-pentylfuran by Aspergillus fumigatus and other respiratory pathogens in vitro and human breath samples. Med Mycol 46:209-215. https://doi.org/10.1080/13693780701753800.

54. Chambers ST, Bhandari S, Scott-Thomas A, Syhre M. 2011. Novel diagnostics: progress toward a breath test for invasive Aspergillus fumigatus. Med Mycol 49:S54-S61. https://doi.org/10.3109/13693786.2010.508187.

55. Chambers ST, Syhre M, Murdoch DR, McCartin F, Epton MJ. 2009. Detection of 2-pentylfuran in the breath of patients with Aspergillus fumigatus. Med Mycol 47:468-476. https://doi.org/10.1080/13693780802475212.

56. Jeleń HH. 2003. Use of solid phase microextraction (SPME) for profiling fungal volatile metabolites. Lett Appl Microbiol 36:263-267. https://doi .org/10.1046/j.1472-765x.2003.01305.x.

57. Acharige MJT, Koshy S, Ismail N, Aloum O, Jazaerly M, Astudillo CL, Koo S. 2018. Breath-based diagnosis of fungal infections. J Breath Res 12:027108. https://doi.org/10.1088/1752-7163/aa98a1.

58. Ahmed WM, Geranios P, White IR, Lawal O, Nijsen TM, Bromley MJ, Goodacre R, Read ND, Fowler SJ. 2018. Development of an adaptable headspace sampling method for metabolic profiling of the fungal volatome. Analyst 143:4155-4162. https://doi.org/10.1039/c8an00841h. 